\title{
Channeling motion of gold nanospheres on a rippled glassed surface
}

\author{
Enrico Gnecco ${ }^{1}$, Pawel Nita ${ }^{1}$, Santiago Casado ${ }^{1}$, Carlos Pimentel $^{2}$, Karine Mougin ${ }^{3}$, Maria \\ Caterina Giordano ${ }^{4}$, Diego Repetto ${ }^{4}$ and Francesco Buatier de Mongeot ${ }^{4}$
}

\author{
1 Instituto Madrileño de Estudios Avanzados en Nanociencia (IMDEA Nanociencia), Campus Universitario de \\ Cantoblanco, Calle Faraday 9, 28049 Madrid, Spain \\ 2 Departamento de Cristalografía y Mineralogía, Universidad Complutense de Madrid, and Instituto de Geociencias \\ (CSIC, UCM), 28040 Madrid, Spain \\ 3 Institut de Science des Matériaux, IS2M-CNRS-LRC 7228, 15 Rue Jean Starcky, 68057 Mulhouse, France \\ 4 Dipartimento di Fisica, Università di Genova, Via Dodecaneso 33, 16136 Genova, Italy
}

\begin{abstract}
Gold nanospheres have been manipulated by atomic force microscopy on a rippled glass surface produced by ion beam sputtering and coated with an ultrathin (10 nm thick) graphitic layer. This substrate is characterized by irregular wavy grooves running parallel to a preferential direction. Measurements in ambient conditions show that the motion of the nanoparticles is confined to single grooves ('channels'), along which the particles move till they are trapped by local bottlenecks. At this point, the particles cross the ripple pattern in a series of consecutive jumps and continue their longitudinal motion along a different channel. Moreover, due to the asymmetric shape of the ripple profiles, the jumps occur in the direction of minimum slope, resembling a ratchet mechanism. Our results are discussed, extending a collisional model, which was recently developed for the manipulation of nanospheres on flat surfaces, to the specific geometry of this problem.
\end{abstract}

Keywords: nanomanipulation, ripples, atomic force microscopy

\section{Introduction}

Since their invention, scanning probe microscopies (SPMs) have rapidly evolved from imaging instruments to operative tools by which individual atoms and molecules can be manipulated at will [1, 2]. When applied to larger systems (e.g., nanoclusters or hepitaxially grown nanoislands), SPM and, more specifically, atomic force microscopy (AFM) manipulation is a formidable tool not only to build artificial nanostructures, but also to investigate the mechanical and chemical interactions at the interface between the nano-objects and the substrate. After the pioneering experiments on $\mathrm{C}_{60}$ and $\mathrm{MoO}_{3}$ islands from the middle 1990s [3, 4], noticeable examples are the controlled manipulation of Au nano-spheres and $\mathrm{MoS}_{2}$ islands by Ritter et al [5,6], the series of experiments by Dietzel et al aimed to define the conditions of occurrence of superlubricity on the nanoscale $[7,8]$, and real-time nanomanipulation experiments inside a scanning electron microscope [9]. However, controlled 
manipulation in terms of lateral or vertical displacement of nano-objects one by one is time consuming and often requires restricted conditions, such as ultra high vacuum or low temperature. In order to fabricate large-scale, functional nanostructures by controlled SPMassisted positioning, different strategies need to be explored. Recently, some of us have showed that AFM allows us to perform fast and controlled manipulation of nanospheres using standard raster scan patterns with variable density of scan lines [10]. Analytical expressions have been derived that relate the angle of deflection $\theta$ of the spheres to the spacing $b$ between consecutive scan lines. This model has been extended to other rigid shapes [11] and supported experimentally on nanorods and flower-shaped islands [12, 13]. However, only atomically flat substrates have been considered in the aforementioned works.

In this paper we explore the influence of the substrate morphology on the manipulation process. A rippled glass surface characterized by irregular grooves is used as a substrate to manipulate $\mathrm{Au}$ nanospheres. The spheres, the diameters of which are below the ripple periodicity, are found to move parallel to the ripples until they get trapped by local impurities or geometric constraints. At this point, a long transverse jump becomes possible. To reduce the friction between particles and substrate and to facilitate the manipulation process, the rippled glass has been covered with an ultrathin graphitic layer. The experimental results are interpreted within our collisional model, extended to the ripple morphology.

\section{Materials and methods}

Self-organized rippled templates were fabricated by ion beam sputtering of low cost soda lime microscope glass slides (Pearl). Ion irradiation was performed in a UHV system with a defocused $\mathrm{Ar}+$ ion beam (5 $\mathrm{N}$ purity) generated from a gridded multiaperture ion source (TECTRA) at an energy of $800 \mathrm{eV}$ and at an incidence angle of $35^{\circ}$, measured with respect to the surface normal. A biased tungsten filament $\left(\mathrm{V}_{\text {bias }}=-13 \mathrm{~V}\right)$, providing electrons by thermoionic emission, was placed close to the extraction grid in order to compensate surface charging due to ion implantation. After an ion dose of about $2.8 \times 10^{19}$ ionscm $^{-2}$, the morphology evolved into a well ordered ripple pattern, which extends uniformly over large areas $\left(\sim \mathrm{cm}^{2}\right.$ scale) with a wavevector mainly oriented parallel to the ion beam projection on the surface [14].

A thin amorphous carbon layer with a thickness of $10 \mathrm{~nm}$ was grown on the ripple template by sputter deposition in a home set-up vacuum chamber. A graphite foil $(0.25 \mathrm{~mm}$ thick, Mateck), attached on a target and placed in front of the sputter gun (TECTRA), was sputtered with an $\mathrm{Ar}^{+}$ion beam at the energy of $4 \mathrm{keV}$. In this condition, a constant flux of about $0.42 \mathrm{nmmin}^{-1}$, monitored by a quartz microbalance, is deposited on the sample. The AFM topography of the template coated with the carbon layer (figure 1(a)) shows that the ripples have a typical length in the range of $4-5 \mu \mathrm{m}$ and a mean height of $50 \mathrm{~nm}$. The lateral periodicity $\lambda$, as obtained from the two-dimensional (2D) autocorrelation function in figures 1(b) and (c), is about $158 \mathrm{~nm}$. 

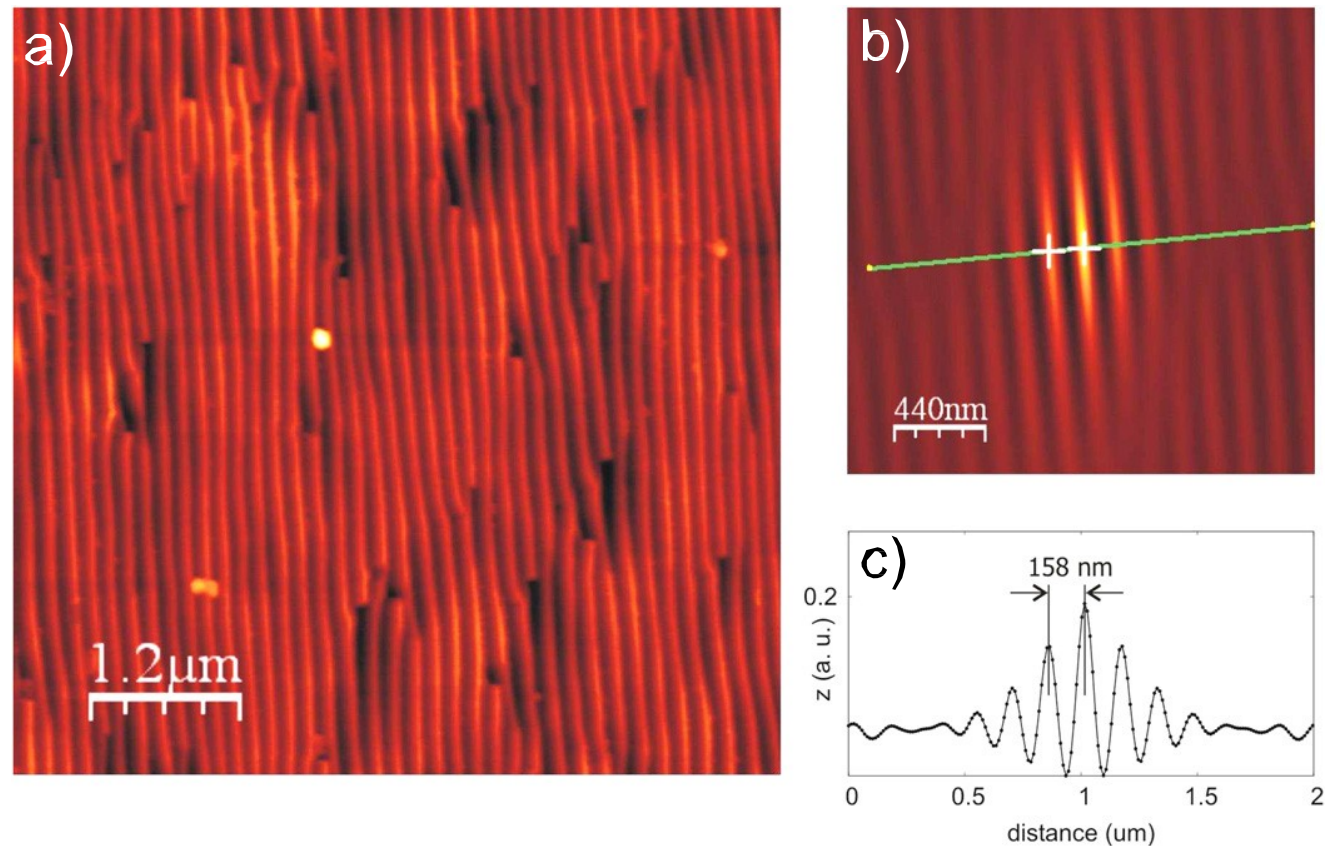

Figure 1. (a) Topography image $\left(5.9 \times 5.9 \mu \mathrm{m}^{2}\right)$ and (b) corresponding 2D autocorrelation pattern. (c) Cross-section of (b) used to estimate the ripples periodicity.

On such a modified substrate, a drop of diluted solution of $\mathrm{Au}$ nanospheres was deposited in ambient conditions. The spheres had a diameter of about $110 \mathrm{~nm}$, as measured by transmission electron microscopy (TEM). After 15 min, the drop was blown off, using dry nitrogen. The manipulation experiments were performed using a Multimode III AFM from Bruker, USA. All measurements were carried out at room temperature and a relative humidity of 25-40\%. SNL- 10D AFM probes from Bruker with nominal resonant frequency $f=18 \mathrm{kHz}$ and spring constant $k_{N}=0.06 \mathrm{~N} / \mathrm{m}$ were used for imaging and nanomanipulation. The manipulation was performed in contact mode, following a zig-zag scan pattern. Data postprocessing was performed using the WSxM [15] and Gwyddion [16] software.

\section{Results}

As seen in figure 1(a), the anisotropic ripple pattern forms an intriguing system of potential channels, similar to river tributaries or railway nodes with bottlenecks and 'dead ends.' In some regions, the channels remain perfectly parallel at distances as long as several $\mu \mathrm{m}$. The $\mathrm{Au}$ nanoparticles deposited on the surface are found to settle exactly in the ripple valleys to minimize their surface energy. No particle was found on the top of the rims separating two consecutive grooves. In figure 2, topography and lateral force maps of the substrate are compared. From the height profile it is seen that the ripple profiles are asymmetric, with a slope of one facet almost twice as large as the other one $\left(\beta=24^{\circ}\right.$ and $\beta^{\prime}=43^{\circ}$ respectively). Due to the anisotropy of the topography profile, forward and backward lateral force sections are not symmetric. Indeed, in both scan directions the lateral force peaks up at the ripples edges, and the peak amplitude is much larger when the edge is reached from the steeper side. From the curvature of the topography profile on the edge, we estimate a radius of curvature of the tip apex in the order of 7-8 nm. These features are important in the interpretation of the measurements discussed below. 

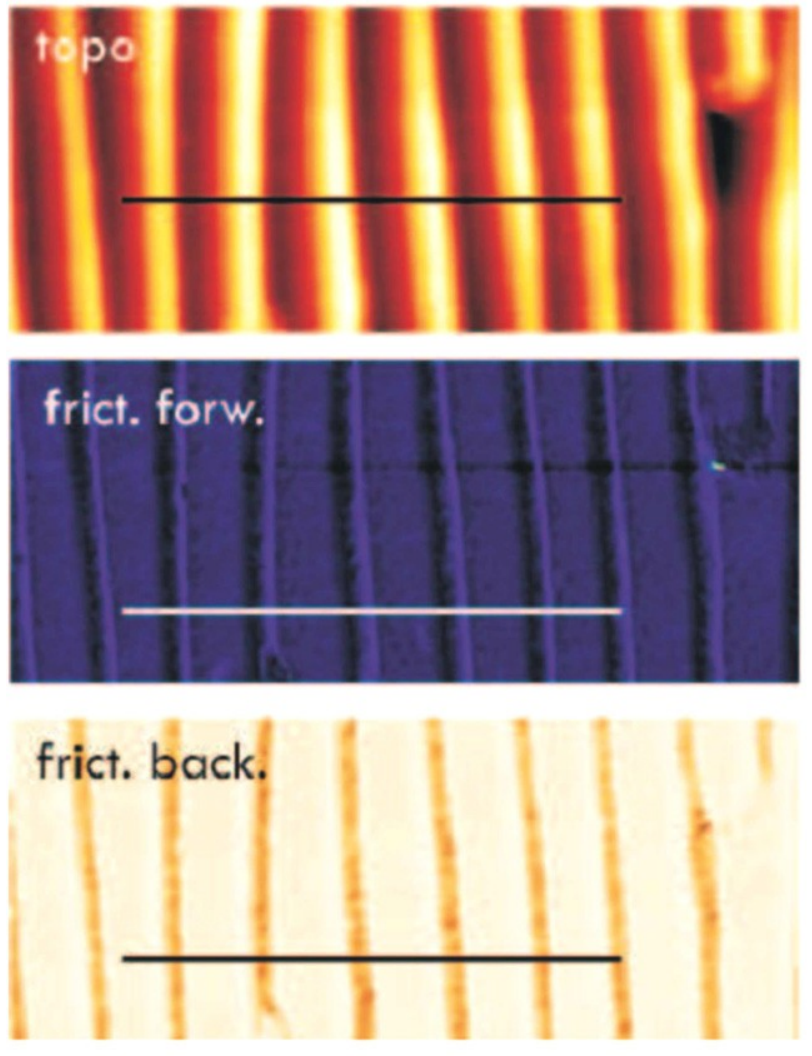

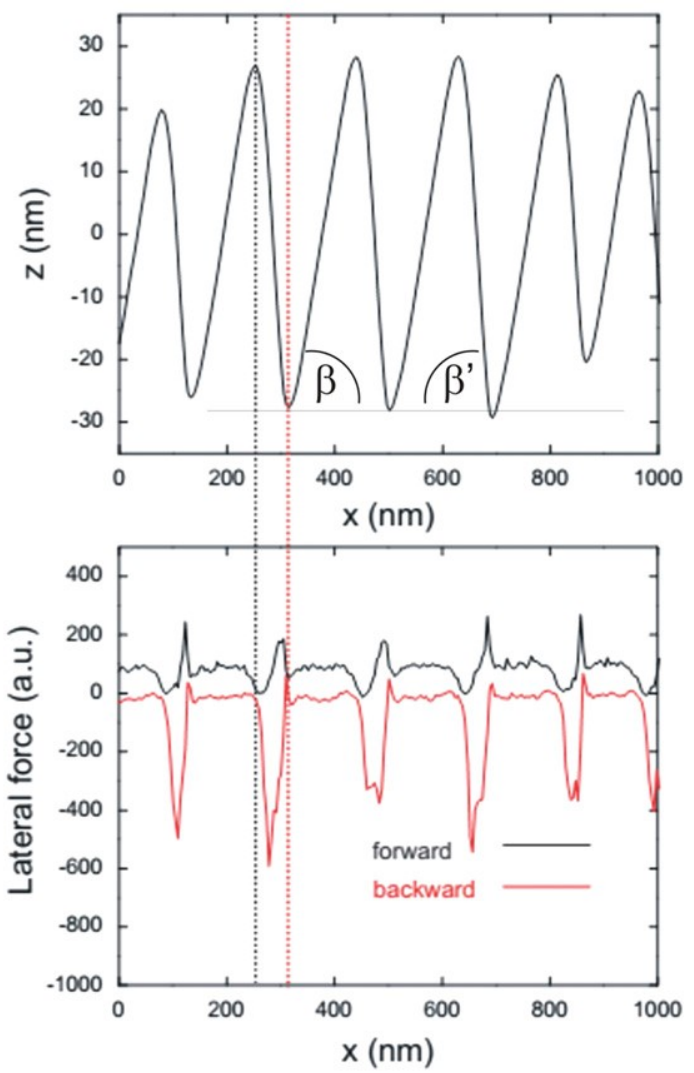

Figure 2. Left panel: AFM topography and corresponding lateral force images (set point $F_{N}=3 \mathrm{nN}$ ). Right panel: Height profile and lateral force signals corresponding to the marked lines.

Nanomanipulation was performed, increasing the normal force FN on the probing tip until a critical value $F_{c}$ was reached, and the Au nanospheres started to move. A representative series of topography images acquired when a sphere was manipulated using a setpoint above $F_{c}$ is shown in figure 3. Here, the fast scan direction (corresponding to the $x$ axis) was chosen almost perpendicular to the ripple orientation (from left to right), and the surface was scanned at a speed of $2.3 \mu \mathrm{ms}^{-1}$. The slow scan direction (y axis) was oriented bottom-up, and the distance between consecutive scan lines was set to $b=4.5 \mathrm{~nm}$. These images clearly reveal the channeling of the nanoparticle during manipulation. Note that the trajectory of the nanoparticle is discontinuous, indicating a stick-slip motion of the sphere along the grooves. This can be attributed to irregular features or impurities in the channel, not resolved by the AFM, which temporarily trapped the particle. In figure 3(a) the nanosphere moved from its initial position up to a crosspoint, where two channels merged into one. In figure 3(c) the sphere continued its longitudinal motion along the main channel till an obstacle (a narrowing point or a surface impurity) was encountered. At this point, the sphere was shifted, crossing four ripple edges to the right, and resumed the previously observed type of motion along a different channel. As shown by the topography profiles corresponding to consecutive scan lines before and after the lateral displacement (figure 4(a)), the particle was displaced transversally by the tip in a series of four consecutive 'jumps' (one per scan line), and not in a single shot. Note that the height difference between the consecutive profiles is com- parable to the sphere diameter, meaning that the tip was almost on top of the particle while manipulating it across the ripples. As seen in figure 4(b), the onset of the particle motion across the grooves is accompanied by a lateral force peak. The peak is much larger at the onset of the first transverse jump (line 2), where the particle is detached from the bottleneck, and adhesion is larger. 

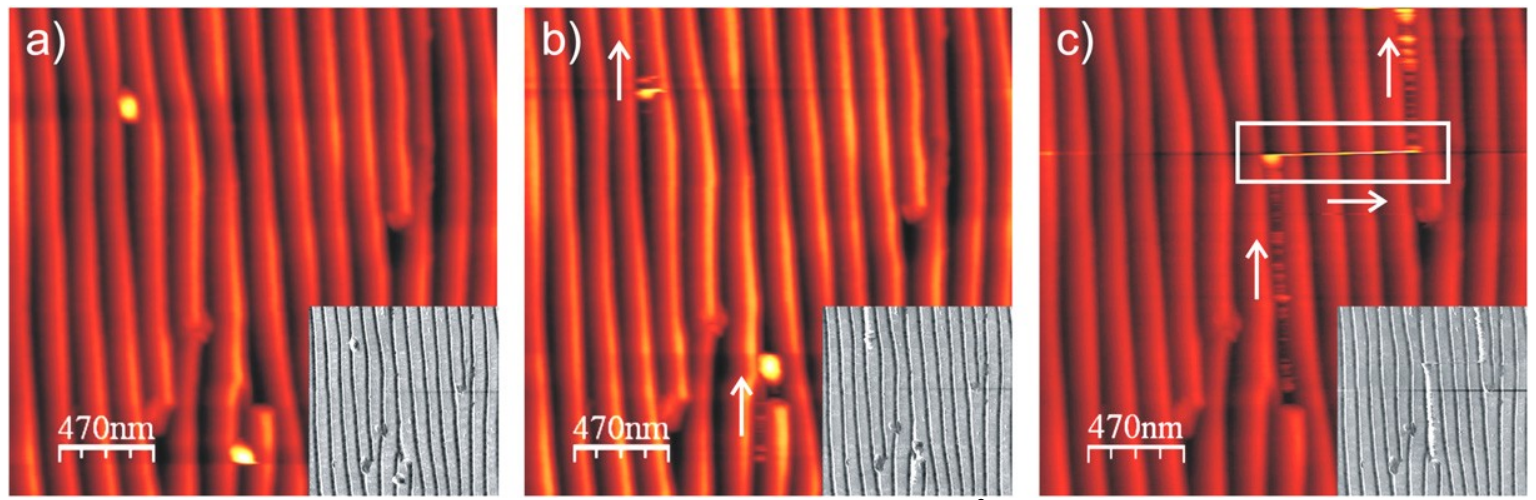

Figure 3. A series of consecutive topography images $\left(2.3 \times 2.3 \mu \mathrm{m}^{2}\right)$ showing the nanomanipulation of two gold nanoparticles. The distance between consecutive scan lines in the zig-zag scan pattern is $4 \mathrm{~nm}$, and the set point $F_{N}=3 \mathrm{nN}$. The arrows indicate the direction of motion of the particles, as inferred from the lateral force images in the insets. The rectangle refers to a region magnified in figure 4, corresponding to multiple jumps of the particle across the ripple pattern.
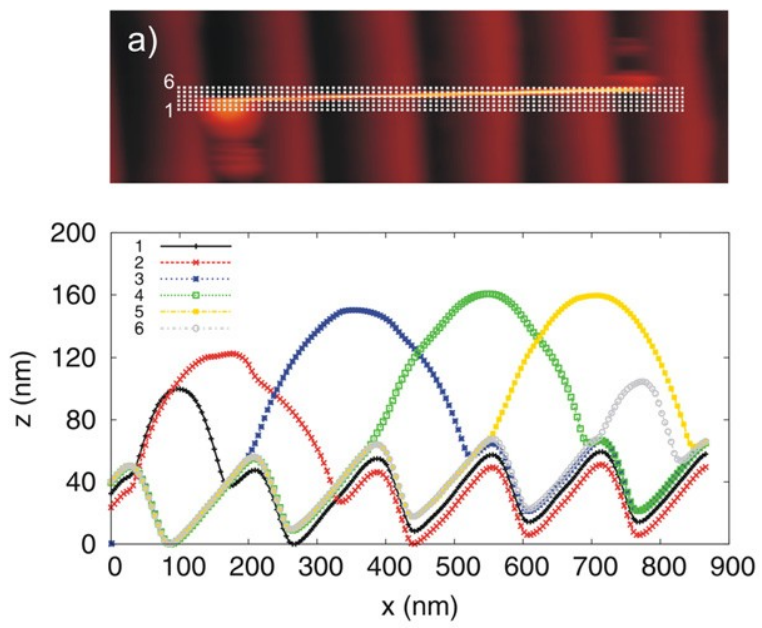
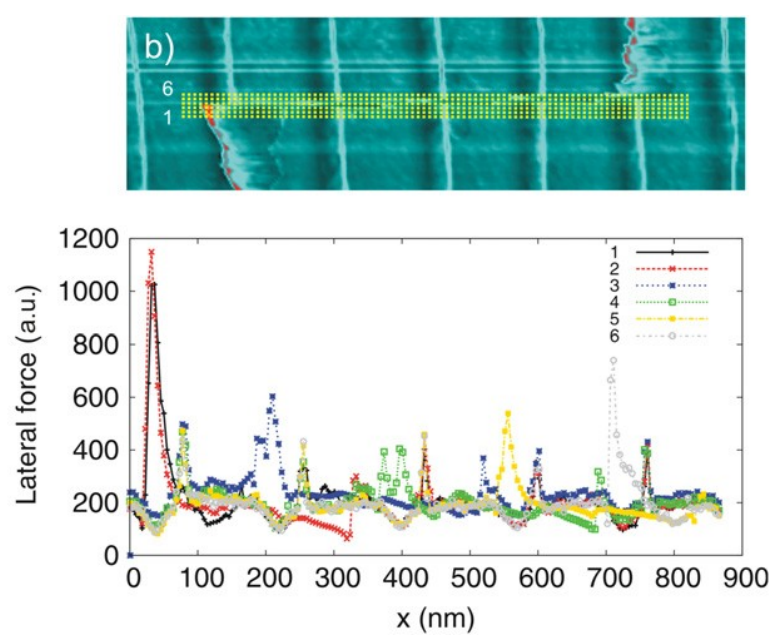

Figure 4. (a) Zoom-in of the rectangular area in figure 3(c) and topography profiles before (1), during (25), and after (6) the multiple jump across the ripple pattern. (b) Corresponding lateral force map and profiles.

Other examples are given in figure 5. Here, three dif- ferent particles were found to jump across seven, one, and two edges, respectively. The total length of these displace- ments was completely uncontrolled. We have also inverted both slow and fast scan directions (right to left and/or top- down, respectively) without changing the orientation of the ripples and, in all cases, the particles were found to jump along the direction of minimum slope, resembling a ratchet mechanism [17]. 


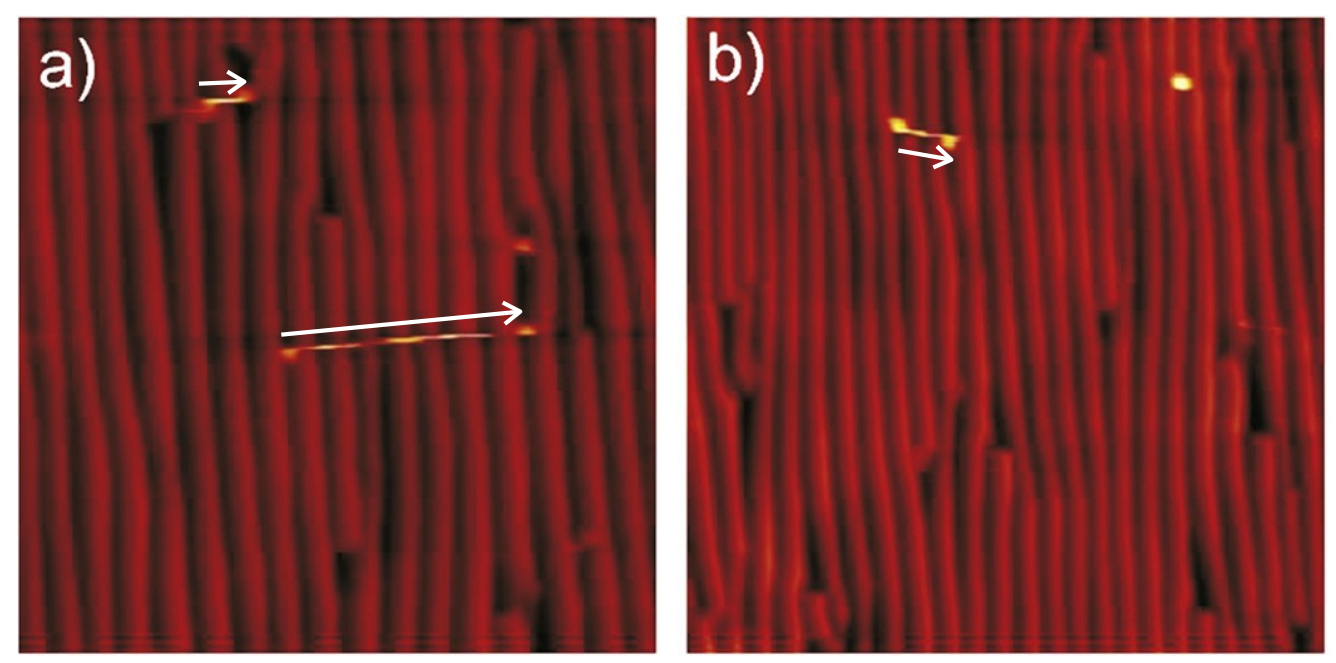

Figure 5. Other examples of nanomanipulation across the ripple pattern. The slow scan direction was oriented (a) upwards, and (b) downwards.

\section{Discussion}

The motion of the nanoparticles can be interpreted by a careful geometric analysis. We distinguish two cases: (i) the particle adheres weakly to the substrate and is displaced along the groove, and (ii) the particle sticks to the substrate and is displaced across the groove only after several impacts along consecutive scan lines.

If a nanosphere adheres only weakly to the substrate, we can gain quantitative insight, extending the model introduced in [10]. There we have proven that, if the sphere lies on a flat surface, its center $O$ advances within one scan line on a distance

$$
\Delta x=-\left(R_{1}+R_{2}\right)\left(\cos \alpha_{0}+\log \tan \frac{\alpha_{0}}{2}\right)
$$

In equation (1), $\alpha_{0}$ is the impact angle (i.e., the angle between the $\mathrm{x}$ axis and the line joining the centers of tip and particle), $R_{1}=R_{p}(1+\sin \gamma) \tan \gamma$, and $R_{2}=R_{p} \cos \gamma$, where $R_{p}=$ $55 \mathrm{~nm}$ is the radius of the nanosphere and $\gamma=22.5^{\circ}$ is the half-angle of the conical tip. (We neglect the tip radius, which, in the present case, is much smaller than $\mathrm{R}_{\mathrm{p}}$.) This model is based on three assumptions: (i) the transfer of momentum between tip and particle occurs only along the line connecting their centers, (ii) the friction between particle and substrate is so high as to prevent further displacement once the particle has lost contact with the tip, and (iii) adhesion between tip and particle is negligible. Following Dietzel et al [18], we assume that these conditions are also verified in contact mode, although an experimental confirmation of the first hypothesis (equivalent to perfect balance of the momentum transferred along the sphere profile by the static friction between tip and particle) is made difficult by the spherical shape of the particles. Note that, according to equation (1), $\Delta x=0$ when the tip hits the lower part of the particle tangentially $\left(\alpha_{0}=90^{\circ}\right)$ and $\Delta x \rightarrow \infty$ in a frontal collision $\left(\alpha_{0}=0^{\circ}\right)$.

In the present problem the $x$ axis is replaced by an axis $x^{\prime}$ tilted by an angle $\beta$. As seen from the geometry in figure 6 , the sphere will reach the top of the ripple if the displacement $\Delta x^{\prime}$ along $x^{\prime}$ is larger than

$$
\Delta x_{c}^{\prime}=\lambda \frac{\sin \beta^{\prime}}{\sin \left(\beta+\beta^{\prime}\right)}-R_{p} \tan \frac{\beta+\beta^{\prime}}{2} \approx 80.7 \mathrm{~nm},
$$

where $\lambda$ is the periodicity of the ripples. 


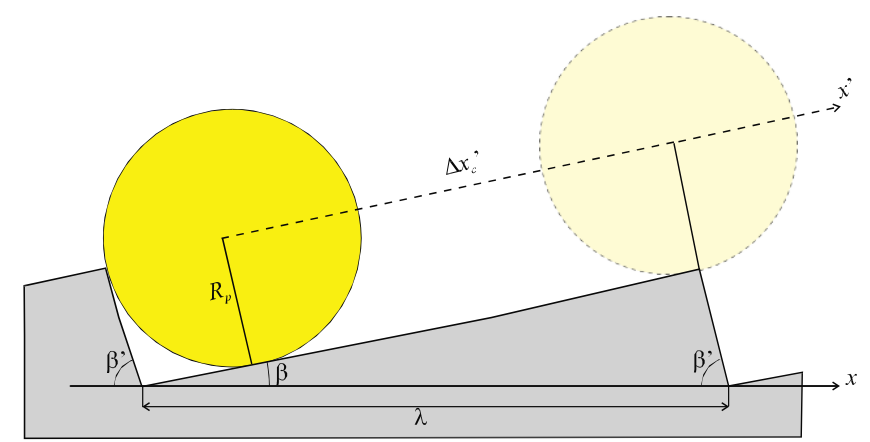

Figure 6. Ripple profile with the nanosphere displaced over it.

Suppose now that the tip hits the sphere after completing its descent along the steepest side of the ripple and starting its rise along the other side. In this case, as shown in the appendix, equation (1) is replaced by

$$
\Delta x^{\prime}=R_{p} \int_{\alpha_{0}}^{\pi / 2}\left(1+\sin (\beta+\gamma) \tan \gamma+\cos \left(\beta^{\prime \prime}+\gamma\right)\right) \frac{\cos ^{2} \alpha}{\sin \alpha} d \alpha,
$$

Where

$$
\beta^{\prime \prime}(\alpha)=\arctan (\cos \alpha \cdot \sin \beta) .
$$

In the appendix we have also estimated that, when $b=4.5 \mathrm{~nm}$, the value of the impact angle $\alpha_{0} \geqslant 71.9^{\circ}$. Note that we assumed a raster scan pattern. With a zig-zag scan pattern we can assume that the formulas are approximately valid in the center of a scan line [10], where the particle manipulated in figure 3 is located. Substituting $\alpha_{0}=71.9^{\circ}$ in equation (2), we conclude that $\Delta x^{\prime}=1.2 \mathrm{~nm}$, which is well below $\Delta x_{c}{ }^{\prime}$. For larger values of $\alpha_{0}, \Delta x^{\prime}$ will be even smaller. Thus, the sphere is only slightly displaced by the tip, and it possibly comes back to the bottom (to minimize the surface energy) when the tip moves forward to the next valley.

A different situation is observed if the particle cannot move along the channel. In this case, a large part of the sphere will be imaged by the tip. The first impact between tip and particle will occur at increasing height along each scan line and, most importantly, at decreasing values of $\alpha_{0}$. Since the tip can hit the sphere while descending the steeper side of the ripple, or even when ascending the previous slope, the formulas derived in the appendix are no longer valid. Although a precise estimation of the critical impact angle in this case requires introducing the AFM feedback in the model, it is not difficult to see that, when the collision tends to be frontal (when almost half of the sphere has been imaged), $\Delta x^{\prime}$ will reach the critical value $\Delta x_{c}{ }^{\prime}$ so that the particle will overcome the barrier and end up in the next channel.

The ratchet mechanism can be also qualitatively understood using figure 6. Assuming that the force exerted by the tip along the $x$ axis is the same while scanning forward and backward, a larger normal force (and, consequently, a larger friction force) will appear when climbing the steepest slope. This means that the value of static friction required to set the particle into motion will be first overcome on the other slope.

It is also interesting to compare our results with the manipulation experiments on similar particles by Paolicelli et al [19]. In that case, the Au nanospheres were found to follow the step edges of a graphite surface or to remain pinned by them. However, no motion across the edges was observed, meaning that the upward and downward directions were essentially equivalent. In our case, the much larger height of the geometric obstacles, comparable to the particles, size, results in two contact areas with different orientations, given by the angles $\beta$ and $\beta^{\prime}$. The two scan directions are no longer equivalent, and the motion across the ripple pattern, although rare, is only seen along the profile with a lower slope. 


\section{Conclusions}

Here, we have conducted an experimental and theoretical analysis on AFM manipulation of gold nanoparticles deposited on a rippled glass surface in ambient conditions. If the ripples are oriented perpendicular to the fast scan direction, the motion of the particles is confined to individual channels unless the displacement is blocked by impurities or geometric constaints. In this case, the particle can cross the ripple pattern transversally and continue its longitudinal motion in a different channel. The total length of the transverse jump is difficult to control, but we observe that a long jump is fractioned in a series of single shift events, each one corresponding to a consecutive scan line. The nanomanipulation process is discussed in terms of a collisional model adapted to the geometry of the problem. A major difficulty that we had to overcome is the variable slope of the line connecting the center of the sphere to the contact point with the tip. With careful geometric modelling of the problem, we have been able to derive a formula which relates the transverse displacement of the sphere in a scan line to the scan pattern and the slope of the ripple. The sphere will not jump if this displacement is less than the width of the ripple side with the lower slope, which is always the case unless the sphere gets trapped by a defect. The method that we have introduced could be extended to other substrates with different morphologies. The ultimate goal is to model the motion of the particles on arbitrarily patterned surfaces using AFM manipulation and to define the best scan path to arrange them in a desired order.

\section{Acknowledgements}

This work was supported by the European COST Action MP1303, the Spanish Ministry of Economy and Competitiveness (MINECO, Project. No. MAT2012-34487), the French Programme 'Nano Espagne,' the Italian Ministry of Foreign Affairs (MAE) in the framework of the Italy-Poland bilateral program, Compagnia di San Paolo, and the University of Genova (UNIGE) in the framework of the PRA2013 project. C.P. is grateful to the Spanish Ministry of Education, Culture and Sports for a FPU grant. AFM observations were carried out at ICTS Centro Nacional de Microscopa Electrónica at the Complutense University of Madrid. We also thank Dr. Carlos Pina and Ms. Patricia Pedraz for helpful comments and technical assistance.

\section{Appendix A. Appendix}

\section{A.1. Manipulation on an inclined plane}

Suppose that a nanosphere is pushed by a conical tip along a plane inclined by an angle $\beta^{\prime \prime}$. Here we assume that the tip is hitting the sphere exactly along the direction of maximum slope so that a cross section of the problem appears as in figure 7(a). The half-angle of the cone is $\gamma$, and the radius of curvature of the tip apex is negligible. Figure 7(c) shows the elliptical intersection between the tip and the plane inclined by an angle $\beta$ passing through the contact point $\mathrm{P}$ between tip and sphere. The major semi-axis of the ellipse can be estimated from the sine law applied to the triangle $\mathrm{VPP}^{\prime}$ as

$$
a=V P^{\prime} \sin \gamma \frac{\cos \gamma}{\cos \left(\beta^{\prime \prime}+\gamma\right)^{\prime}}
$$

where 


$$
V P^{\prime}=R_{P} \frac{1+\sin (\beta+\gamma)}{\cos \left(\beta^{\prime \prime}+\gamma\right)} .
$$

The minor semi-axis b can be obtained from figure 7(d) usingthe notations in figure 7(b) ( $\mathrm{C}$ is the center of the ellipse, at a distance q from the axis of the cone, and $\mathrm{p}$ is the radius of the circular section of the cone passing through $\mathrm{C}$ and parallel to the xy plane):

$$
b=\sqrt{(p+q)(p-q)},
$$

where $p$ and $q$ are obtained from figure 7(b) as

$$
\begin{gathered}
q=a \cos \beta^{\prime \prime}-V P^{\prime} \sin \gamma \\
p=V P^{\prime} \sin \gamma+a \sin \beta^{\prime \prime} \tan \gamma .
\end{gathered}
$$

The minor radius of curvature of the ellipse $R_{1}$ " $=b^{2} / a$ plays the role of 'tip' radius in the manipulation of the sphere along the $x^{\prime} y^{\prime}$ plane. Using equations (A.1)-(A.3) after some simplifications, we get

$$
R_{1}^{\prime \prime}=R_{P}\left(1+\sin \left(\beta^{\prime \prime}+\gamma\right)\right) \tan \gamma,
$$

whereas the relation

$$
R_{2}^{\prime \prime}=R_{P} \cos \left(\beta^{\prime \prime}+\gamma\right)
$$

for the effective sphere radius in the planar problem is simply obtained from figure 7(a).

a)

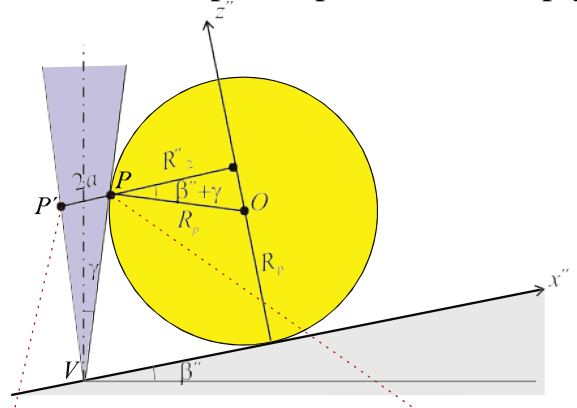

b)

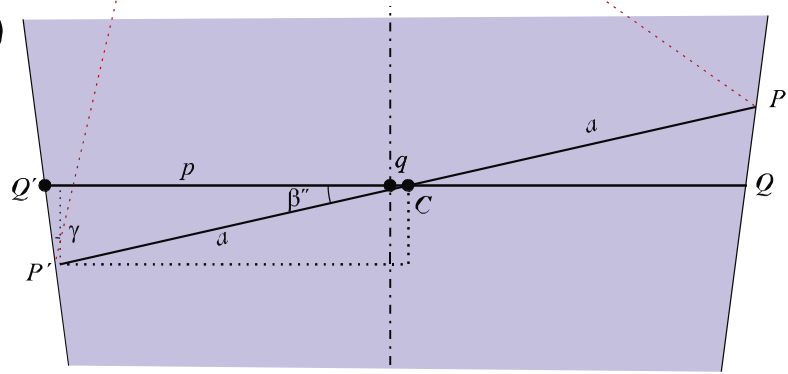

c)

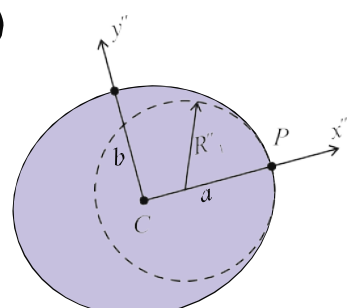

d)

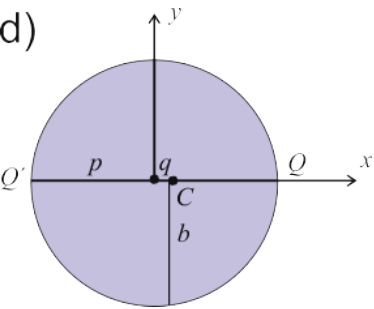

Figure 7. Side view of a conical tip pushing a nanosphere up an inclined plane. 
As a next step, we need to find a relation between $\beta^{\prime \prime}$ and the angle of inclination $\beta$ of the plane with respect to the $x$ axis. From figure 8 it can be seen that $\beta^{\prime \prime}$ depends on the impact angle $\alpha$, as

$$
\tan \beta^{\prime \prime}=\cos \alpha \sin \beta
$$

The infinitesimal variation of the coordinate $x^{\prime}$ of the sphere center, when the angle of contact between tip and sphere, $\alpha$, increases by $d \alpha$ is [10]:

$$
d x^{\prime}=R \frac{\cos \alpha}{\tan \alpha} d \alpha
$$

leading to the expression (2) in the main text.

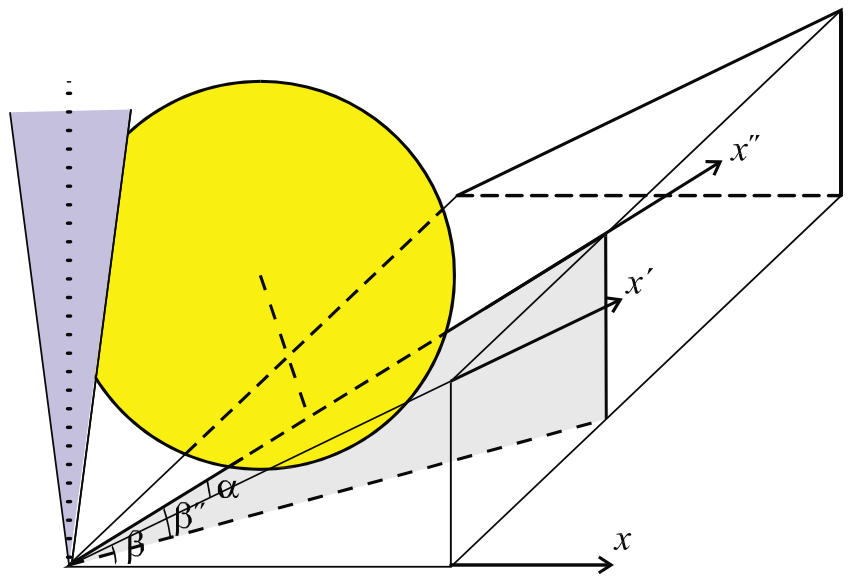

Figure 8. Geometric relation between the angles $\beta, \beta^{\prime \prime}$, and $\alpha$ defined in the text.

\section{A.2. Minimum impact angle}

The minimum value of the impact angle $\alpha_{0}$ is obtained as follows. Figure 9(a) shows a situation in which the tip almost touches the sphere while moving along the $x^{\prime}$ axis. In the next scan line, at a distance $b$ along $y^{\prime}$, the tip will touch the sphere with the minimum possible value of $\alpha_{0}$. In figure 9(a) we define the length

$$
c=\frac{R_{P}}{\tan \left(45^{\circ}-\gamma / 2\right)}-b=R_{P} \frac{1+\tan (\gamma / 2)}{1-\tan (\gamma / 2)}-b .
$$

This length can be related to $\alpha_{0}$ using figure 9(b):

so that

$$
c=\left(R_{1}^{\prime \prime}+R_{2}^{\prime \prime}\right) \sin \alpha_{0}
$$

$$
\left(R_{1}^{\prime \prime}+R_{2}^{\prime \prime}\right) \sin \alpha_{0}=R_{P} \frac{1+\tan (\gamma / 2)}{1-\tan (\gamma / 2)}-b
$$

Combining equation (A.7) with the relations (A.4), (A.5), and (A.6), one can estimate $\alpha 0$ numerically. With the values of $\beta, \gamma, R_{p}$, and $b$ of our problem, we obtain $\alpha_{0}=71.9^{\circ}$. 
a)

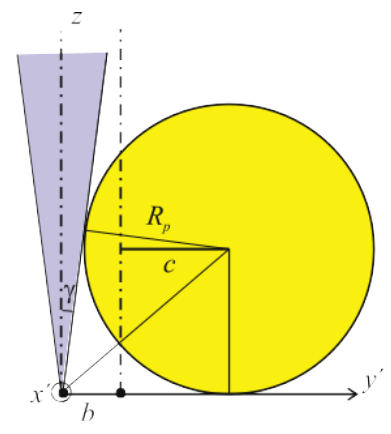

b)

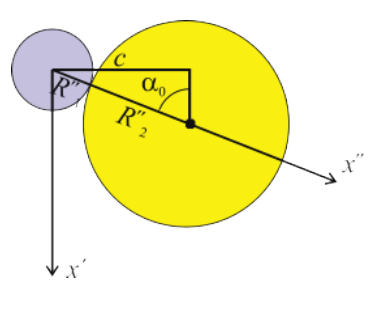

Figure 9. (a) Front view and (b) top view used to determine the minimum impact angle between tip and sphere (in the $x^{\prime} y^{\prime}$ plane).

\section{References}

[1] Eigler D M and Schweizer E K 1990 Nature 344524

[2] Sugimoto Y, Pou P, Custance O, Jelinek P, Abe M, Perez R and Morita S 2008 Science 322 413-7

[3] Lüthi R, Meyer E, Haefke H, Howald L, Gutmannsbauer W and H J G 1994 Science 266 1979

[4] Sheehan P E and Lieber C M 1996 Science 2721158

[5] Ritter C, Heyde M, Schwarz U D and Rademann K 2002 Langmuir 18 7798-803

[6] Ritter C, Heyde M, Rademann K and Schwarz U D 2005 Phys. Rev. B 71085405

[7] Dietzel D, Ritter C, Monninghoff T, Fuchs H, Schirmeisen A and Schwarz U D 2008 Phys. Rev. Lett. 101125505

[8] Dietzel D, Feldmann M, Schwarz U D, Fuchs H and Schirmeisen A 2013 Phys. Rev. Lett. 111235502

[9] Fahlbusch S, Mazerolle S, Breguet J M, Steinecker A, Perez R and Michler J 2005 J. Mat. Proc. Tech. 167371

[10] Rao A, Gnecco E, Marchetto D, Mougin K, Schönenberger M, Valeri S and Meyer E 2009 Nanotechnology 20115706

[11] Gnecco E 2010 Beilstein J. Nanotech. 158

[12] Gnecco E, Rao A, Mougin K, Chandrasekar G and Meyer E 2010 Nanotechnology 21 215702

[13] Nita P, Casado S, Dietzel D, Schirmisen A and Gnecco E 2013 Nanotechnology 21215702

[14] Toma A, Buatier de Mongeot F, Buzio R, Firpo G, Bhattacahrryya S, Boragno C and Valbusa U 2005 Nuclear Instruments and Methods B 230 551-4

[15] Horcas I, Fernández R, Gómez-Rodríguez J M, Colchero J, Gómez-Herrero J and Baro A M 2007 Rev. Sci. Instrum. 78013705

[16] Necas D and Klapetek P 2012 Cent. Eur. J. Phys. 10181

[17] Jung P, Kissner J G and Hänggi P 1996 Phys. Rev. Lett.76 3436

[18] Dietzel D, Feldmann M, Herding C, Schwarz U D and Schirmeisen A 2010 Trib. Lett. 39 273

[19] Paolicelli G, Rovatti M, Vanossi A and Valeri S 2009 Appl. Phys. Lett. 95143121 